\title{
What determines saccade timing in sequences of coordinated eye and hand movements?
}

\author{
Roger W. Remington • Shu-Chieh Wu • Harold Pashler
}

Published online: 5 March 2011

(C) Psychonomic Society, Inc. 2011

\begin{abstract}
In action sequences, the eyes are generally fixated ahead of the stimulus being responded to, overlapping the processing of adjacent stimuli. What determines when the saccade to the next item is initiated and what processing is overlapped remain controversial. Saccade initiation has been linked directly to the difficulty of the underlying processing on the fixated item or to a rhythmic timer whose period is adjusted to the difficulty of the context. We compared the effects of item and context difficulty when subjects made speeded choice responses to five visual stimuli arrayed linearly. Item difficulty was manipulated by varying stimulus-response compatibility, creating contexts (sequences) whose average item difficulty differed. Results showed that saccade initiation time reflected item difficulty, not context difficulty, with evidence that saccade initiation was delayed until central processing was completed. We discuss the implications of these results for the control of saccades and processing in action sequences.
\end{abstract}

R. W. Remington $(\bowtie)$

School of Psychology,

University of Queensland,

St Lucia, QLD 4072, Australia

e-mail: r.remington@uq.edu.au

S.-C. Wu

San Jose State University and NASA Ames Research Center, Moffett Field, CA, USA

H. Pashler

University of California at San Diego,

San Diego, CA, USA
We spend much of our time engaged in activities that involve coordinated eye and hand movements. For example, when writing or editing a document, we make a series of fixations punctuated by saccades from one word to the next, interspersed with hand movements for typing and mousing. Despite decades of study on a range of eye-hand tasks, it is still not known how these overt eye and hand movements are coordinated with the underlying processing. One clue to this coordination is that with preview of upcoming items, the eyes are fixated ahead of the item to which the response is being made (e.g., Inhoff \& Gordon, 1997; Land \& Lee, 1994; Salthouse, 1986; Vickers, 1992). Preview also leads to interresponse intervals (IRIs) that are shorter than response times in isolation (Pashler, 1994; Salthouse, 1986), suggesting that looking ahead takes advantage of parallelism in the human cognitive architecture to overlap portions of the stimulus processing for successive actions, creating a smooth, fluid sequence. Initiating a saccade from an item prior to the completion of its processing creates the potential that its remaining processing will overlap with that of the next item. The question that has yet to be answered is what events in the processing stream determine when the eyes saccade to the next item.

The question can be made more concrete by considering the possibilities for scheduling saccade initiation within a central bottleneck cognitive architecture. In central bottleneck theory, processing unfolds in three serial, independent stages: stimulus encoding (SE), which leads to the identification of a stimulus; response selection (RS), which associates the identified stimulus with an arbitrary response; and response execution (RE), which outputs the selected response (Pashler, 1984; Sternberg, 1969; Welford, 1952). Stimulus encoding and response execution for multiple stimuli can proceed in parallel with each other 
and with response selection. Response selection requires central processing resources, so that response selection for multiple stimuli must be executed in series. Voluntary saccades have been shown to require limited-capacity central resources (Pashler, Carrier, \& Hoffman, 1993), suggesting that the central demands of the saccade must be done in series with the response selection (i.e., central demands) for the manual response.

Figure 1 shows three possible ways to coordinate saccade initiation with ongoing stimulus processing. A few studies have shown evidence for saccade initiation prior to response selection (Salthouse \& Ellis, 1980; Salthouse, Ellis, Diener, \& Somberg, 1981; Sanders \& van Duren, 1998). As is shown in Fig. 1a, completing saccade central processing prior to response selection allows response selection on stimulus $N$ (e.g., $\mathrm{RS}_{2}$ ) to be done in parallel with response execution on $N-1$ (e.g., $\mathrm{RE}_{1}$ ) and stimulus encoding on $\mathrm{N}+1$ (e.g., $\mathrm{SE}_{3}$ ), creating the shortest possible sequence of processing stages in central bottleneck theory: $\mathrm{SE}_{1}+\sum \mathrm{RS}_{i=1}-{ }_{N}+\mathrm{RE}_{N}$. A similar model by Pashler (1994), without explicit treatment of saccades, produced good qualitative fits to observed IRIs in a sequence task with preview of two or more upcoming items.

In general, however, fixation duration (the interval between saccades) is sensitive to factors that affect processing at or subsequent to response selection: lexical frequency (Inhoff \& Jian, 1992; Salthouse, 1986), motor difficulty (e.g., Inhoff, Rosenbaum, Gordon, \& Campbell, 1984), and stimulus-response compatibility (Wu \& Remington, 2004; Wu, Remington, \& Pashler, 2004). These findings are consistent with saccade initiation after the completion of response selection. As is shown in Fig. 1b, such a central bottleneck model is conservative in that it overlaps only $\mathrm{RE}_{N}$ with $\mathrm{SE}_{N+1}$, leaving the central processor idle during $\mathrm{SE}_{N+1}$. As a result, it does not yield minimum sequence completion times according to central bottleneck theory.

The models above are instances of a general class of process control accounts of saccade initiation, which link saccade initiation directly to the completion of some portion of processing on the currently fixated stimulus (e.g., Reichle, Pollatsek, Fisher, \& Rayner, 1998; Williams \& Pollatsek, 2007). In contrast, global estimation accounts posit only an indirect link with stimulus processing. They assume that fixation duration is estimated from recent processing history and is used to set an interval timer, which then determines when the next saccade is made (Hooge \& Erkelens, 1998; Nuthmann, Smith, Engbert, \& Henderson, 2010; Remington, Lewis, \& Wu, 2006). Because global estimation accounts look beyond the individual item level, saccade initiation can be adapted to optimize some aspect of overall sequence execution. For example, Fig. 1c illustrates an adaptive
Fig. 1 Figure 1 illustrates the Three hypotheses for how saccade intervals are determined. $\mathrm{SE}_{N}=$ stimulus encoding on item $N, \mathrm{RS}_{N}=$ response selection on $N, \mathrm{SAC}_{N}=$ the saccade to item $N$, and $\mathrm{RE}_{N}=$ response execution on $N$. a Saccaderelated central processing is completed prior to response selection. SE and RE are fully overlapped with RS stages. b Saccade-related central processing is completed after response selection. Only SE and RS stages overlap. c An interval timer (line at bottom) triggers a saccade at regular intervals. To illustrate the adaptive character of the timer, the interval $(i)$ is determined by a running mean of previous durations for response selection
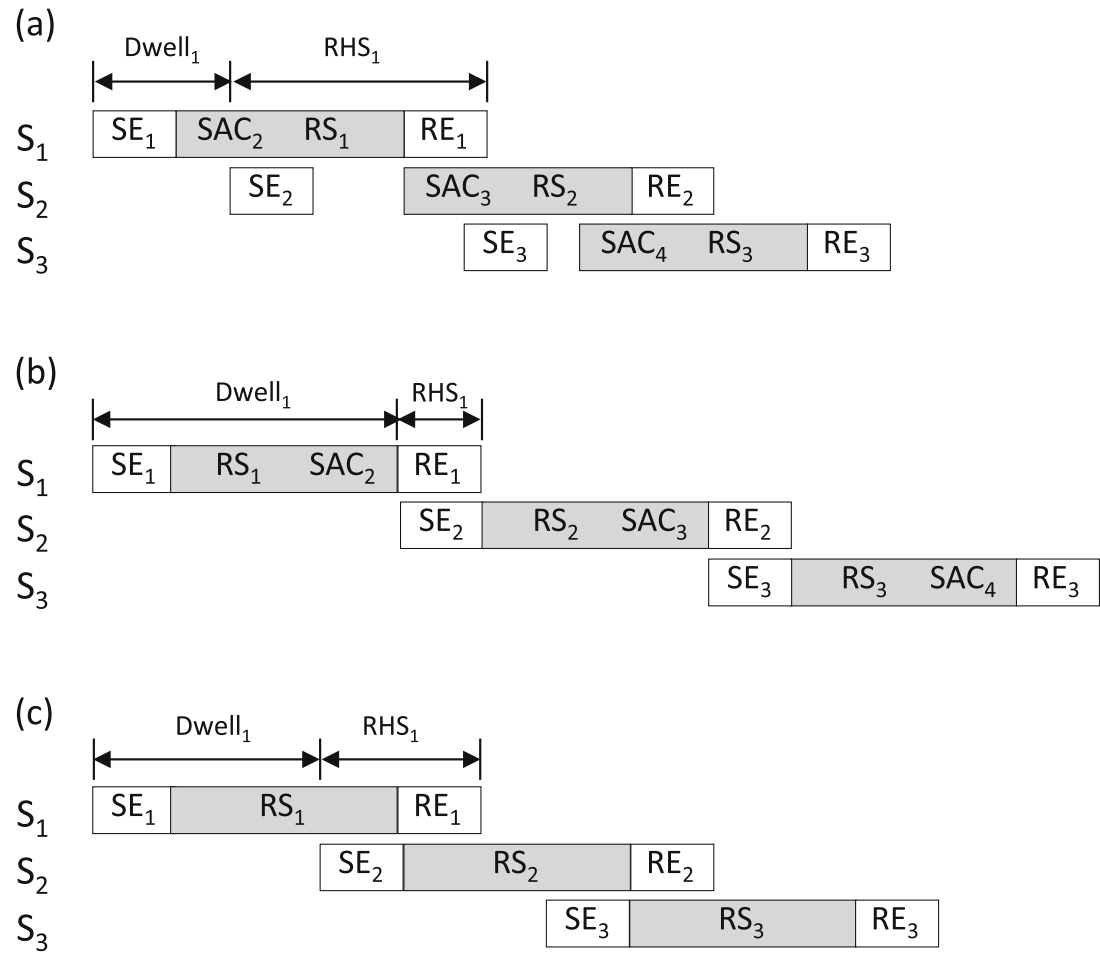

Timing Pulse: $i_{n}=\left({ }_{k} \Sigma S_{k<n}\right) /(n-1)$ 
rhythmic timer for saccade initiation whose interval is adjusted to match the average of previous response selection durations. Accordingly, $\mathrm{SE}_{N+1}$ finishes at the same time as $\mathrm{RS}_{N}$ (Remington et al., 2006), producing an efficient schedule equivalent to Fig. 1a.

Global estimation accounts are attractive because they derive saccade initiation times from consideration of a rational agent adapting to task demands. In addition, entrainment by rhythmic patterns has been shown to affect attention and perceptual judgments (e.g., McAuley \& Jones, 2003). In action sequences, saccade intervals and IRIs tend to converge on the same value after a few items (Wu \& Remington, 2004), producing a saccade-response rhythm. However, for an interval timer using global estimates, item difficulty should affect only the saccade interval for subsequent items, making it difficult to account for the longer fixation durations of difficult items observed in studies of reading and visual search (e.g., Reichle et al., 1998). Hybrid models overcome this by allowing cognitive processing to inhibit timer-determined saccade initiation (e.g., Nuthmann et al., 2010).

Adaptive timers make an interesting, untested prediction. If the estimate for the saccade timer interval is derived from the recent history of processing, the timing of the saccade on $\mathrm{S}_{N}$ should be influenced by the difficulty of the preceding $N-1$ items. If so, an easy stimulus in the context of more difficult stimuli should show longer saccade intervals (dwell times) than when in an easy context. Conversely, the saccade interval for a difficult item among easier items should be less than required to complete response selection, leaving some central processing to be done in the postsaccade interval. Thus, whereas process control accounts predict effects of item difficulty on saccade initiation, timer models predict strong effects of context difficulty, with longer postsaccade intervals for hard items in easy contexts.

We investigated the determinants of saccade timing by having subjects execute a sequence of choice responses to a linearly arrayed set of stimuli (see, e.g., Pashler, 1994; Wu \& Remington, 2004; Wu et al., 2004). Item difficulty was manipulated by varying stimulus-response compatibility, which should affect response selection. Contexts of varying difficulty were created by populating sequences with stimuli of predominately compatible or incompatible mappings. A saccade to the next stimulus $\left(\mathrm{S}_{N+1}\right)$ before a response to the current one $\left(\mathrm{S}_{N}\right)$ yields two measures of interest, shown in Fig. 1. Dwell measures the saccade interval: the total time from first fixating a stimulus until initiating a saccade to the next. Release-hand span (RHS) measures the postsaccade interval: the time from the saccade away from a stimulus to its response. The RHS is the interval during which processing of $\mathrm{S}_{N}$ overlaps with that of $\mathrm{S}_{N+1}$.
The central bottleneck model shown in Fig. $1 \mathrm{~b}$ predicts that dwell should vary directly with the duration of response selection, without affecting RHS. Neither measure should be affected by the difficulty of the context. For the adaptive timer model shown in Fig. 1c, saccade initiation is determined by prior context. Hence, the model predicts that dwell will be affected by context difficulty, not by stimulus difficulty. The sensitivity to context leads to the critical trade-off predicted when stimulus difficulty differs from that of the overall context. According to the timer model shown in Fig. 1c if the difficulty of $\mathrm{RS}_{2}$ is less than that of the average context, as in Fig. $2 \mathrm{a}, \mathrm{RHS}_{2}$ will be shorter than average; if the difficulty of $\mathrm{RHS}_{2}$ is greater, as in Fig. $2 b, \mathrm{RHS}_{2}$ will be longer than average, with potential effects on $\mathrm{S}_{3}$.

\section{Method}

Subjects made a series of speeded choice responses to five stimuli arrayed linearly across a CRT screen. Each stimulus consisted of a $2 \times 2$ matrix containing two, three, or four identical digits and one or more fillers completing the total

(a)

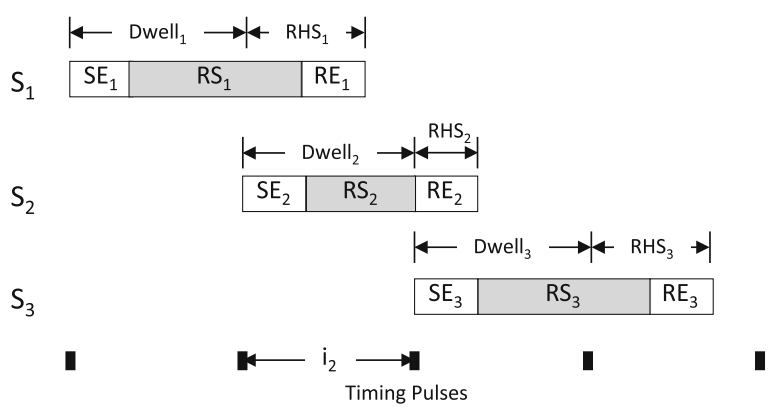

(b)

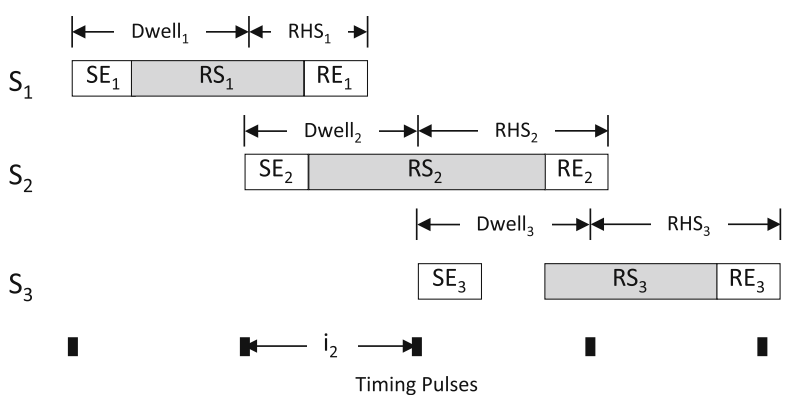

Fig. 2 Figure 2 shows the Dependent measures and the trade-offs predicted by an adaptive interval timer. Dwell $l_{N}=$ the total time looking at item $N$, equivalent to the interval between saccades.; RHS $_{N}=$ releasehand span (RHS) for item $N$ : the duration of post-saccade processing, defined as the interval from the saccade away from $N$ to the response to $N$. The pulse timer is shown below each figure. a $\mathrm{RS}_{2}$ is reduced, causing $\mathrm{RHS}_{2}$ to be reduced, with no effects on subsequent items. b $\mathrm{RS}_{2}$ is lengthened, causing $\mathrm{RHS}_{2}$ to be lengthened, as well as $\mathrm{RHS}_{3}$. If difficulty levels are held constant, saccade intervals ( $d$ well) will adapt, converging on the new RS duration 
of four. The task was to quickly indicate how many digits were present in a given stimulus. Response selection difficulty was manipulated by varying compatibility between the number of digits (reported dimension) and digit identity (unreported). For compatible stimuli, the number of digits was the same as the digit identity (e.g., 2-2-X-X, 3-3-3-X). For incompatible stimuli, the digit identity conflicted with the number (e.g., 4-4-X-X, 2-2-2-X). Context was varied by creating sequences that differed in the number of compatible and incompatible items. Pure sequences were homogeneous with respect to difficulty, all hard (incompatible) or all easy (compatible). Mixed sequences included one or two stimuli from the nondominant condition. The experiment was conducted in four sections, two predominantly easy, two predominantly hard, counterbalanced between subjects for order of presentation. Subjects Nineteen undergraduate students were recruited from local universities and community colleges near the NASA Ames Research Center. All reported normal or corrected-to-normal vision. They participated for class credit or monetary compensation.

Apparatus and stimuli Separate Pentium 4 computers controlled the experimental sequence and eye movement recording. Eye movements were monitored with a headmounted video-based eye-tracking system (Applied Sciences Laboratory, Model 501) sampling at $120 \mathrm{~Hz}$ with a spatial precision of approximately $0.5^{\circ}$ of visual angle. Experiments were carried out in a quiet, well-lit room, with subjects seated approximately $60 \mathrm{~cm}$ from a 21-in. CRT display with a $70-\mathrm{Hz}$ refresh.

The stimulus display consisted of a row of five $2 \times 2$ matrices $\left(0.34^{\circ}\right.$ in height $)$ across the middle of the display, spaced approximately $5.5^{\circ}$ apart, presented at a luminance of $11.7 \mathrm{~cd} / \mathrm{m}^{2}$. The digits " 2 ," "3," or " 4 " appeared in two, three, or four cells of the matrix. Remaining cells were filled with "X." Subjects pressed the V, B, or N key, respectively, to indicate whether there were two, three, or four digits present. On easy trials (E), the number of digits was compatible with the digits themselves; on hard trials $(\mathrm{H})$, it was incompatible.

Procedure A total of 384 trials were presented in four blocks of 96 trials each, preceded by 24 practice trials. In easy blocks, the four sequences were: EEEEE, EHEEE, EEHEE, and HEEHE. In hard blocks, the four sequences were the mirror of the those in the easy block: НHНHН, $\mathrm{HEHHH}, \mathrm{HHEHH}$, and EHHEH. Block difficulty alternated, with block order counterbalanced across subjects.

Each trial began with the presentation of a white fixation cross $\left(0.3^{\circ}\right)$ in the center of the display. After $1,000 \mathrm{~ms}$, the fixation cross was erased, and a small filled square $\left(0.34^{\circ}\right)$ appeared at the leftmost stimulus position. Subjects were instructed to fixate the small square and maintain fixation until the stimuli were presented. The small square remained for $500 \mathrm{~ms}$, followed by a blank interval of $500 \mathrm{~ms}$, after which the sequence was presented. Eye movement recording began the moment the small square appeared over the location of the leftmost item and ended after the subject had responded to the rightmost stimulus. A calibration procedure was administered before each block to maintain accuracy of recordings. The characters were erased after the subject had responded to the rightmost character. The next trial began following an intertrial interval of $1,000 \mathrm{~ms}$. Subjects were instructed to respond to each item as quickly and accurately as they could and not to group their responses.

Manual responses and fixations were recorded for each item, timed from the onset of the stimulus sequence. A fixation was defined as movement velocity below $30 \%$ or movement acceleration below $-3000 \%$, on the basis of horizontal ( $x$-axis) movements only. A detected fixation was assigned to the nearest stimulus position. Dwell on a stimulus was calculated by summing all contiguous individual fixations on a designated target region that were equal to or greater than $75 \mathrm{~ms}$. End of dwell was determined by the first fixation of any duration on a different item. Once a fixation on an item ended, subsequent fixations on that item were considered regressions. Regressions and other anomalous fixations were omitted from analysis.

\section{Results and discussion}

Five of the 19 subjects were excluded: 4 because of malfunctions in the eye tracking, resulting in lost or corrupted data, and 1 because the pattern of eye movements was too erratic. For the remaining 14 subjects, we analyzed only trials that contained a clear sequence of left-to-right eye movements, resulting in further pruning of approximately $1 \%$ of the trials. Only correct responses whose fixation to response interval was less than 2,000 ms were analyzed.

Probability correct was .982 for easy (compatible) items and .984 for hard (incompatible) items (.983 overall), which yielded a significant effect of difficulty (arcsine transform of percent correct), $F(1,13)=7.1, p<.05$, but no effect of context, $F<1$.

A preliminary analysis showed significant effects of stimulus position on dwell and RHS, but no interactions with context or item difficulty. Consequently, subsequent analyses were collapsed over position. Calculation of RHS included only stimuli in which the eyes moved prior to the response (positive RHS). Unless otherwise specified, all analyses of variance have degrees of freedom $=1,13$.

Analysis of fixation patterns revealed that subjects made an average of 1.74 fixations per item; $53 \%$ of the stimuli were fixated once, $31 \%$ twice. Mean first-fixation durations 
were 590 and $250 \mathrm{~ms}$ for one-fixation and two-fixation cases, respectively. Total dwell time increased linearly from one to four fixations per stimulus, with a slope of $115.4 \mathrm{~ms}$ per fixation $\left(R^{2}=.987\right)$.

Does context or stimulus processing determine saccade initiation time? Means for dwell and RHS as a function of context and item difficulty in mixed-difficulty sequences are shown in Table 1. For RHS, an analysis of variance revealed no effects of context, $F<1$, or item difficulty, $F<1$. For dwell, the effect of item difficulty was significant, $F=97$, $p<.001$, the effect of context did not approach significance, $F<1$, but the interaction of context and item difficulty was significant, $F=16, p<.001$. As is shown in Table 1 , this interaction was due to elevated dwell on easy items in a hard context. Dwell on hard stimuli was unaffected by context. The elevation of dwell for easy items in a hard context, but not for hard items in an easy context, is consistent with some hybrid rhythmic timer accounts, such as CRISP (e.g., Nuthmann et al., 2010), in which saccades are initiated according to a timing pulse but delayed if cognitive processing is not complete. As shown below, however, elevated dwell on easy items in a hard context was due to the effects of the immediately preceding stimulus, not the context.

Previous tests of process control and rhythmic timer accounts have focused on the duration of a single fixation, usually the first (e.g., Williams \& Pollatsek, 2007). Analysis of our first-fixation durations showed a significant effect of item difficulty (easy $=436 \mathrm{~ms}$, hard $=454 \mathrm{~ms}$ ), $F=15.2, p<$ .01 , and no effect of context, $F<1$. Unlike dwell, the interaction of context and item difficulty failed to reach significance, $F=2.1, p<.20$. An analysis of single-fixation cases showed the same pattern as the first-fixation analysis, including the absence of a significant context $\times$ item difficulty interaction $(p>.15)$. An analysis of mean RHS on single-fixation cases showed no significant effects of context or difficulty, all $F_{\mathrm{s}}<1$. Our main findings, then, are robust across three metrics of fixation duration.

\section{Was central processing complete prior to saccade initiation?} We sought converging evidence for process control accounts by examining whether response selection was

Table 1 Means (standard deviation) (in milliseconds; with standard deviations) for dwell and release-hand span as a function of context and stimulus difficulty. Easy = compatible stimulus--response mappings., Hard = incompatible stimulus--response mappings

\begin{tabular}{lllll}
\hline & Dwell & & \multicolumn{2}{l}{ Release-Hand Span } \\
\hline Context & Stimulus & & & \\
& Easy & Hard & Easy & Hard \\
Easy & $635(133)$ & $694(149)$ & $385(134)$ & $393(144)$ \\
Hard & $659(128)$ & $685(125)$ & $397(121)$ & $398(125)$ \\
\hline
\end{tabular}

completed prior to saccade initiation. In the timer model shown in Fig. 1c, increasing the duration of $\mathrm{RS}_{N}$ would yield a saccade prior to completion of $\mathrm{RS}_{N}$, leaving unfinished central processing in $\mathrm{RHS}_{N}$ that could create resource conflicts when overlapped with $\mathrm{S}_{N+1}$ processing. In the central bottleneck model shown in Fig. 1b, increased $\mathrm{RS}_{N}$ duration would have no effect on $\mathrm{S}_{N+1}$ processing, since RHS $_{N}$ should contain only response execution processes. With no resource conflicts, overlapped processing should proceed in parallel. Analyses of adjacent pairs of stimuli for effects of $\mathrm{S}_{N}$ on $\mathrm{S}_{N+1}$ showed modest effects of $\mathrm{S}_{N}$ difficulty on $\mathrm{S}_{N+1}$. When $\mathrm{S}_{N}$ was hard, $\mathrm{S}_{N+1}$ showed elevated mean RHS for both easy and hard $\mathrm{S}_{N+1}, F=8.5, p<.05$, elevated mean dwell for easy $\mathrm{S}_{N+1},(F=4.9, p<.05$, and elevated first-fixation duration for easy $\mathrm{S}_{N+1},(F=5, p<.05$. Dwell and first-fixation duration for hard $\mathrm{S}_{N+1}$ were unaffected by the difficulty of $\mathrm{S}_{N}, F<1$. The effect of the previous stimulus accounts for the effect of context on dwell for easy stimuli reported above; an easy stimulus in a hard context was always preceded by a hard item, while, in an easy context, it was more often preceded by another easy item.

Three pieces of evidence suggest that the observed prioritem effect was not due to unfinished central processing. First, when we examined instances of hard-easy pairs in which the response to the hard item was made before the saccade, ensuring that central processing on the hard item was completed prior to saccade initiation, dwell on the subsequent easy stimulus was actually longer $(671 \mathrm{~ms})$ than when the response was made after the saccade $(630 \mathrm{~ms})$. Second, we found no correlation between the duration of the RHS of the hard stimulus and the dwell on the subsequent easy stimulus $(R=.06)$. If central processing had occurred during the RHS, it should have interfered with central processing on the easy stimulus, increasing dwell. Since unfinished central processing would have to be longer than the stimulus encoding of the easy item to produce central interference, we reran the correlation with only those RHSs above the median (325 ms). The correlation was still low $(R=.1)$. Third, the mean dwell on $\mathrm{S}_{N+1}$ when the RHS of the hard $\mathrm{S}_{N}$ was above the median ( $325 \mathrm{~ms}$ ) was $638 \mathrm{~ms}$, as compared with $622 \mathrm{~ms}$ when below the median, which was not significant, $F<1$. Thus, we found no evidence that the elevated dwell following a hard item was due to unfinished central processing.

What then explains the prior-item effect? Hybrid timer models, such as CRISP (e.g., Nuthmann et al., 2010), could account for the effect of a difficult $\mathrm{RS}_{N}$ on $\mathrm{S}_{N+1}$ dwell because the saccade is delayed until central processing is completed. This could delay the subsequent saccade if, for example, the regular timing pulse on $\mathrm{S}_{N+1}$ was missed on some trials. This, however, cannot account for the lengthened $\mathrm{RHS}_{N+1}$. In our view, the most likely explanation is that subjects adapted their cognitive processing following 
an incompatible stimulus, for example, by adding verification operations to response selection (elevating dwell on $\mathrm{S}_{N+1}$ ), raising response activation thresholds (elevating $\mathrm{RHS}_{N+1}$ ), or other measures to prevent an erroneous response to the more strongly associated number value. Summed dwell was affected most, since those cases with greater difficulty in suppressing the incorrect compatible response were associated with an increased number of fixations.

\section{Conclusions}

Our results provide new evidence from a novel paradigm for two important claims about the control of saccades from one item to the next in tasks requiring coordinated hand and eye movements. First, saccade initiation is determined principally by the state of processing on the currently fixated item (see, e.g., Rayner \& Pollatsek, 1981; Williams $\&$ Pollatsek, 2007), not by a rhythmic timer. This links the overt behavior in action sequences to the schedule of underlying processing operations. Second, subjects complete response selection before initiating the saccade to the next item, as previously observed in reading (Inhoff \& Jian, 1992; Salthouse, 1986) and other sequential tasks (e.g., Inhoff et al., 1984). Our results can be accommodated by hybrid timer models if, as in CRISP, they provide process control mechanisms that delay saccade initiation until central processing is completed. The multiple fixations on stimuli that comprised our summed dwell suggest that not all endogenous saccades may be so dependent on response selection, although the mechanisms involved in those saccades is unclear. Nonetheless, taken together with existing data, our findings support the conclusion that dependency on the completion of central processing of the fixated item is a general property of item transitions in sequential tasks.

Author note This work was supported by Grant FA9550-07-1-0356 from the Air Force Office of Scientific Research to Roger Remington, Shu-Chieh Wu, and Harold Pashler, and by Grant DP066672 from the Australian Research Council to Roger Remington.

\section{References}

Hooge, I. T. C., \& Erkelens, C. J. (1998). Adjustment of fixation duration in visual search. Vision Research, 38, 1295-1302.

Inhoff, A. W., \& Gordon, A. M. (1997). Eye movements and eyehand coordination during typing. Current Directions in Psychological Science, 6, 153-157.

Inhoff, A. W., \& Jian, W. (1992). Encoding of text, manual movement planning, and eye-hand coordination during copytyping. Journal of Experimental Psychology: Human Perception and Performance, 18, 437-448.
Inhoff, A. W., Rosenbaum, D. A., Gordon, A. M., \& Campbell, J. A. (1984). Stimulus-response compatibility and motor programming of manual response sequences. Journal of Experimental Psychology: Human Perception and Performance, 10, 724-733.

Land, M. F., \& Lee, D. N. (1994). Where we look when we steer. Nature, 369, 742-744.

McAuley, J. D., \& Jones, M. R. (2003). Modeling effects of rhythmic context on perceived duration: A comparison of interval and entrainment approaches to short-interval timing. Journal of Experimental Psychology: Human Perception and Performance, 29, 1102-1125.

Nuthmann, A., Smith, T. J., Engbert, R., \& Henderson, J. M. (2010). CRISP: A computational model of fixation durations in scene viewing. Psychological Review, 117, 382-405.

Pashler, H. (1984). Processing stages in overlapping tasks: Evidence for a central bottleneck. Journal of Experimental Psychology: Human Perception and Performance, 10, 358-377.

Pashler, H. (1994). Overlapping mental operations in serial performance with preview. The Quarterly Journal of Experimental Psychology, 47A, 161-191.

Pashler, H., Carrier, M., \& Hoffman, J. (1993). Saccadic eyemovements and dual-task interference. The Quarterly Journal of Experimental Psychology, 46A, 51-82.

Rayner, K., \& Pollatsek, A. (1981). Eye-movement control during reading: Evidence for direct control. The Quarterly Journal of Experimental Psychology, 33A, 351-373.

Reichle, E. D., Pollatsek, A., Fisher, D. L., \& Rayner, K. (1998). Toward a model of eye movement control in reading. Psychological Review, 105, 125-157.

Remington, R. W., Lewis, R., Wu, S.-C. (2006). Scheduling mental operations in a multiple-response sequence: Modeling the effects of a strategy to minimize variance in the timing of saccades. In D. Fum, F. del Missier, \& A. Stocco (Eds.), Proceedings of the 7th International Conference on Cognitive Modeling. Trieste, Italy. April 2-6, 2006

Salthouse, T. A. (1986). Perceptual, cognitive, and motoric aspects of transcription typing. Psychological Bulletin, 99, 303-319.

Salthouse, T. A., \& Ellis, C. L. (1980). Determinants of eye-fixation duration. The American Journal of Psychology, 93, 207-234.

Salthouse, T. A., Ellis, C. L., Diener, D. C., \& Somberg, B. L. (1981). Stimulus-processing during eye fixations. Journal of Experimental Psychology: Human Perception and Performance, 7, 611623.

Sanders, A. F., \& van Duren, L. L. (1998). Stimulus control of visual fixation duration in a single saccade paradigm. Acta Psychologica, 99, 163-176.

Sternberg, S. (1969). Discovery of processing stages: Extensions of Donders' method. Acta Psychologica, 30, 276-315.

Vickers, J. N. (1992). Gaze control in putting. Perception, 21, 117132.

Welford, A. T. (1952). The 'psychological refractory period' and the timing of high-speed performance - a review and a theory. British Journal of Psychology, 43, 2-19.

Williams, C. C., \& Pollatsek, A. (2007). Searching for an O in an array of Cs: Eye movements track moment-to-moment processing in visual search. Perception \& Psychophysics, 69, $372-381$.

Wu, S.-C., \& Remington, R. W. (2004). Coordination of component mental operations in a multiple-response task. In S. N. Spencer (Ed.), Proceedings of the eye tracking research and applications symposium 2004. New York: ACM SIGGRAPH.

Wu, S.-C., Remington, R. W., \& Pashler, H. (2004). Coordination of component mental operations in sequences of discrete responses. In K. Forbus, D. Gentner, \& T. Regier (Eds.), Proceeding of the twenty-sixth annual conference of the cognitive science society (pp. 1476-1481). Mahwah: Erlbaum. 